\title{
Elevated IL-13Ra2 in intestinal epithelial cells from ulcerative colitis or colorectal cancer initiates MAPK pathway
}

\author{
Debasmita Mandal, M.S. ${ }^{*}$ and Alan D. Levine, Ph.D. , ${ }^{\ddagger}$ [Senior Author] \\ "Department of Pathology, 10900 Euclid Avenue, Case Western Reserve University School of \\ Medicine, Cleveland, Ohio 44106-4952 \\ ‡Departments of Medicine, Surgery, Pharmacology, and the Case Comprehensive Cancer \\ Center, 10900 Euclid Avenue, Case Western Reserve University School of Medicine, Cleveland, \\ Ohio 44106-4952
}

\begin{abstract}
Chronic inflammation in ulcerative colitis (UC) is a sizeable risk factor for colorectal cancer (CRC). Interleukin-13 (IL-13) is elevated in the UC mucosa and may induce dysregulated signaling in neighboring intestinal epithelial cells (IEC) and thus function as a tumorogenic cytokine. Expression of IL-13 receptor chains on IEC obtained from control or chronically inflamed mucosa and colonic tumors was quantified by flow cytometry and immunoblot. IL-13Ra 1 and IL-13Ra 2 expression is significantly increased on IEC from UC and CRC patients compared to control and CD subjects. Purified IEC from these subjects and cell lines expressing varying ratios of IL-13Ra 1 and a 2 chains were stimulated with IL-13 in vitro to investigate by immunoblot the activation of the STAT6 and MAPK signaling pathways. Despite similarly elevated receptor expression in UC and CRC, IL-13 does not activate the STAT6 or MAPK pathways in UC, while in colonic tumors only the STAT6 pathway is activated. Using neutralizing antibodies and cell lines expressing a range of surface densities for IL-13Ra 1 and IL-3Ra2, we demonstrate that IL-13Ra2 serves a dual role, initiating MAPK signaling at low concentrations and as an inhibitory, decoy receptor at high IL-13Ra2 to IL-13Ra 1 ratios. Thus IL-13Ra 2 is both a decoy receptor and induces MAPK signal transduction, depending on its relative expression and the local concentration of IL-13, which together modulate the balance and intensity of the signaling pathways initiated in UC and CRC.
\end{abstract}

\section{Keywords}

IL-13 Receptor; Colorectal Cancer; Ulcerative Colitis; STAT6; MAP Kinase

\section{Introduction}

Colorectal cancer (CRC) is the second leading cause of cancer death in the United States among men and women (1). Patients with colonic inflammatory bowel disease (IBD), such as ulcerative colitis (UC), are at higher risk of developing $\mathrm{CRC}$, which increases with the duration and severity of disease (2). As it is believed that the inflammation process itself is responsible for transformation of the intestinal epithelium, insight into the pathogenesis of UC-associated CRC will come from studying the immune cells found in the chronic

\footnotetext{
Address correspondence to: Alan D. Levine, Ph.D., Department of Medicine, Case Western Reserve University School of Medicine BRB426, 10900 Euclid Avenue, Cleveland, Ohio, 44106-4952. Phone: 01-216-368-0342, Fax: 01-216-368-1674,

alan.levine@case.edu.

Conflicts of interest: No conflicts of interest exist.
} 
inflammatory microenvironment and their products, such as chemokines and cytokines, which play a role in tumor development and progression in several cancers (3).

Inflammation associated colon cancer has also been observed in many natural and genetically engineered murine models of colitis $(4,5)$. We reported in the IL-10-/- model that as inflammation becomes chronic, mucosal expression of the cytokine IL-13 increases (6). Older mice at 35 weeks of age develop dysplasia at the inflamed focal lesions, and neutralizing IL-13 partially alleviates inflammation (6) and prevents the appearance of lowgrade dysplasia. Inflammation in another model of colitis, which resembles human UC, is induced by topical application of oxazolone and is mediated by natural-killer T (NKT) cells that also produce IL-13 (7). Furthermore, it was reported that upon polyclonal activation of lamina propria mononuclear cells from involved tissue of UC patients containing CD4+ cells bearing NKT cell marker (CD161) secrete greater quantities of IL-13 as compared with equivalent cells from control or Crohn's disease (CD) patients, another form of IBD $(8,9)$. Thus dysregulation of IL-13 production might contribute to intestinal inflammation in UC patients and possibly predispose an inflamed mucosa to dysplastic and neoplastic transformation.

IL-13, a $17 \mathrm{kDa}$ cytokine secreted by activated T cells, NKT cells, human airway smooth muscle cells, renal cell carcinomas, and Hodgkin's Reed-Sternberg tumor cells, is involved in allergic inflammation, fibrosis, goblet cell hyperplasia, and tumor cell growth (10). In airway and intestinal epithelial cell lines IL-13 regulates activities that may contribute to carcinogenesis, such as inducing the goblet cell product trefoil factor-3 (11), which is overexpressed in various cancers and associated with tumor invasion, resistance to apoptosis, and metastasis, and downregulating expression of E-cadherin and carcinoembryonic antigen (12). IL-13 also stimulates expression of CD44v6 and CD44v3 (13), which confer metastatic properties to rat carcinoma cells and are expressed in CRC and adenomatous polyps. IL-13 can induce aberrant expression of activation-induced cytidine deaminase in colonic epithelial cells and thus links inflammation, somatic mutations, and colorectal cancer development (14).

It is widely believed that signal transduction in an IL-13 targeted cell is mediated by two receptor complexes, one that stimulates a response and another inhibitory receptor. On hematopoietic cells the IL-13 receptor is composed of a $65-70 \mathrm{kDa}$ IL-13Ra 1 chain with low binding affinity to IL-13, the shared $\gamma_{C}$ cytokine chain, and the $140 \mathrm{kDa}$ IL-4Ra chain (10). In non- hematopoietic cells that lack $\gamma_{\mathrm{C}}$, the receptor consists of IL-13Ra 1 and IL-4Ra (15). IL-13Ra 1 is also an accessory component of the IL-4 signaling complex $(16,17)$. The inhibitory IL-13Ra 2 chain is a $55-60 \mathrm{kDa}$ protein with high affinity for IL-13 and a short cytoplasmic tail (17 amino acids). Thus, the strength and character of an IL-13 signal are proposed to be mediated by the balance between IL-13Ra 1 and IL-13Ra 2 expression $(18,19)$. One report proposes that IL-13 signals through IL-13Ra2 to stimulate an activator protein (AP-1) variant containing c-jun and fra-2, which initiates transcription at the transforming growth factor $\beta 1$ (TGF- $\beta 1$ ) promoter and thereby induces TGF- $\beta 1$ production (20). Therefore, IL-13Ra 2 might act as a signaling receptor as well as a decoy receptor.

Tumorigenesis can be envisaged as a recurring series of mutations and selective cell growth. Chronic inflammation may provide both mutagens and growth factors to feed these recurring cycles for progression of oncogenesis. We investigated the contribution in UCassociated CRC of one such growth factor, IL-13, which is in excess in UC. In this report we demonstrate increased expression of IL-13 receptor chains (IL-13Ra 1 and IL-13Ra2) in the epithelium from UC patients and CRC lesions. Our study reveals distinct IL-13-induced signaling patterns through the STAT6 and MAPK pathways in epithelial cells from UC and 
CRC patients, not observed in IEC from control and CD patients. Using neutralizing antibodies to IL-13Ra1, IL-13Ra2-transfected cells, and by sorting for cells expressing various ratios of IL-3Ra 1 and IL-3Ra2, we report that at low concentrations IL-3Ra2 signals through the MAPK pathway and at higher concentrations functions as a decoy receptor for both STAT6 and MAPK.

\section{Materials and Methods}

\section{Isolation of Intestinal Epithelial Cells}

Surgical specimens were obtained from patients undergoing bowel resection at University Hospitals Case Medical Center. Primary intestinal epithelial cells (IECs) were isolated from control and diseased donors (21). Colon specimens were thoroughly rinsed in HBSS

(Cambrex, Walkersville, MD), and cut into strips that were washed at room temperature for $30 \mathrm{~min}$ in $1.5 \mathrm{mg} / \mathrm{mL}$ dithiothreitol (Fisher Scientific, Fair Lawn, NJ) to remove the mucus and then gently stirred in $1 \mathrm{mmol} / \mathrm{L}$ EDTA (Sigma-Aldrich, St Louis, MO) for $60 \mathrm{~min}$, which causes the crypts to detach. Three washes were collected and the crypts were treated with $3 \mathrm{mg} / \mathrm{ml}$ dispase (Roche, Indianapolis, IN) and $1 \mathrm{mg} / \mathrm{ml}$ deoxyribonuclease I (Worthington, Lakewood, NJ) in $15 \mathrm{ml}$ RPMI 1640 (Cambrex) for $30 \mathrm{~min}$ at $37^{\circ} \mathrm{C}$. The sample was vortexed for $10 \mathrm{~s}$ at $5 \mathrm{~min}$ intervals. Cells were washed twice with RPMI 1640 and purified by separation over a 50\% Percoll (GE Healthcare Biosciences, Piscataway, NJ) gradient, centrifuged at $1500 \mathrm{rpm}$ for $20 \mathrm{~min}$. IECs that equilibrated at the interface were collected and washed twice in RPM1 1640.

\section{Isolation of colon carcinoma cells}

Non-necrotic, malignant tissue was thoroughly rinsed in HBSS and minced into 1 to $3 \mathrm{~mm}$ fragments. The fragments were placed in $20 \mathrm{ml}$ HBSS containing 5 units $/ \mathrm{ml}$ hyaluronidase (Type IV-S, Sigma-Aldrich), $2 \mathrm{mg} / \mathrm{ml}$ collagenase (CLS type III, Worthington), $0.3 \mathrm{mg} / \mathrm{ml}$ deoxyribonuclease (Type I, Worthington), and $2.5 \%$ penicillin-streptomycin-fungizone mixture (Lonza, Walkersville, MD) and stirred at low speed for $60 \mathrm{~min}$ at room temperature (22). Cells were collected by gentle aspiration of the supernatant and centrifugation at 1500 rpm for $5 \mathrm{~min}$ in Sorvall T 6000D centrifuge, and further purified by fractionation over a $50 \%$ Percoll gradient.

\section{Flow Cytometry}

$1 \times 10^{6}$ cells were incubated with monoclonal antibodies to IL-13Ra 1 (Clone \# 116730), IL-13Ra2 (Clone \#83834), IL-4Ra (Clone \#25463), or isotype control antibodies (all at 10 $\mu \mathrm{g} / \mathrm{ml}$ from R \& D Systems) for $30 \mathrm{~min}$ on ice. The cells were washed twice in PBS containing $5 \%$ FCS, incubated with PE-labeled goat anti-mouse $\mathrm{F}(\mathrm{ab})_{2}$ fragment $(10 \mu \mathrm{g} / \mathrm{ml}$; Dako, Carpinteria, CA) for $30 \mathrm{~min}$ on ice, washed, and fixed with $2 \%$ formaldehyde. At least 10,000 events were analyzed using the Coulter XL-MCL Flow Cytometer (Beckman Coulter, Inc, Fullerton, CA) and quantified using Winlist software (Verity Software House, Topsham, ME). Median fluorescence signals were calculated for each test $\left(\mathrm{F}_{t}\right)$ and background sample $\left(\mathrm{F}_{\mathrm{b}}\right)$. Data is expressed as specific fluorescence $\left(\mathrm{F}_{\mathrm{sp}}\right)$, which is $\mathrm{F}_{\mathrm{t}}-\mathrm{F}_{\mathrm{b}}$.

\section{Immunoblotting}

Whole cell lysates were prepared in RIPA buffer (50 mM Tris (Fisher Scientific), pH 7.5, $150 \mathrm{mM} \mathrm{NaCl}$ (Fisher Scientific), 1\% NP-40 (Sigma-Aldrich), 0.5\% sodium deoxycholate (Sigma-Aldrich), $0.1 \%$ SDS (Roche)) supplemented with 1\% protease inhibitor and 1\% phosphatase inhibitor cocktail (Sigma-Aldrich). Protein concentration was determined using the BCA protein assay reagent (Pierce, Rockford, IL). Equal amounts of protein $(20 \mu \mathrm{g})$ were resolved by SDS-PAGE on a 10\% gel under reducing conditions, electrotransferred to 
nitrocellulose membranes (Invitrogen Life Technologies), blocked, and incubated sequentially with primary antibodies (same as those described for flow cytometry) overnight at $4^{\circ} \mathrm{C}$ and HRP-conjugated goat anti-mouse IgG (Santa Cruz Biotechnology, CA) diluted (1:1000) for $1 \mathrm{~h}$ at room temperature (23). Signal was detected with West Pico Supersignal (Pierce) and BioMax MR Film (Kodak, Rochester, NY).

\section{Stimulation of IEC, HT-29.19A, and U937}

Freshly isolated IEC $\left(5 \times 10^{6}\right.$ cells) were rested for $2 \mathrm{~h}$ in DMEM at $37^{\circ} \mathrm{C}$, resuspended in $100 \mu 1$ of fresh DMEM, and equilibrated at $37^{\circ} \mathrm{C}$ for $5 \mathrm{~min}$, before being stimulated with IL-13 (R \& D) at $37^{\circ} \mathrm{C}$, followed by addition of $100 \mu \mathrm{l} 2 \times$ Laemmli buffer to stop the reaction. Samples were boiled for $10 \mathrm{~min}$, stored at $-20^{\circ} \mathrm{C}$, and later analyzed via immunoblotting for phospho-specific and total STAT6, ERK, JNK, and p38 (Cell Signaling). HT-29.19A and U937 cells were rested $4 \mathrm{~h}$ at $37^{\circ} \mathrm{C}$ in media lacking FCS, and where indicated pre-incubated with $60 \mu \mathrm{g} / \mathrm{ml}$ of polyclonal anti-IL-13Ra 1 (R \& D Systems) and monoclonal anti-IL-4Ra for an hour at $37^{\circ} \mathrm{C}$, and stimulated with IL-13. The reaction was stopped, boiled, and analyzed by immunoblot as described above.

\section{Cytokine differentiation of HT-29.19A cells to increase IL-13Ra2 expression}

IL-13 (20 ng/ml) and TNF-a (20 ng/ml; R \&D Systems) were added to 70-75\% confluent HT-29.19A cells for $72 \mathrm{~h}$. Cytokine-treated and untreated cells were sorted with a triplelaser BD Aria sorter (San Jose, CA) and gated into two subpopulations, those expressing no detectable IL-13Ra2 and others with various IL-13Ra2 expression as indicated in the figure.

\section{Statistical Analysis}

Data is presented as mean and standard error of the mean. Differences between IL-13Ra1, IL-13Ra2, and IL-4Ra expression among the various disease conditions were examined using analysis of variance (ANOVA) followed by the least significant difference (LSD) comparison procedure (SAS - Statistical Analysis System, Version 9.1, Cary, NC). All tests are two-tailed and p-values $\leq 0.05$ are considered to be statistically significant.

\section{Results}

\section{Increased expression of IL-13 receptor subunits IL-13Ra1 and IL-13Ra2 in IEC from inflamed UC and involved CRC lesions}

Since chronic inflammation in UC patients increases the probability of colonic tumorigenesis, we hypothesized that IL-13-induced responses in the UC epithelium may be dysregulated and that the IL-13 receptor would be preferentially expressed on UC IEC. Constitutive expression of the individual IL-13 receptor subunits, IL-13Ra1, IL-13Ra2, and IL-4Ra, was examined on IEC isolated from control and diseased patients (UC, CD, and CRC) by flow cytometry (Figure 1). Contaminating lamina propria mononuclear cells represented less than $2 \%$, as assessed by staining for the common leukocyte antigen CD45, while greater than $98 \%$ of the IEC stained positive for an epithelial cell marker with antiEpCam antibodies (data not shown). Expression of the IL-13Ra 1 and IL-13Ra2 subunits is increased on a subset of both UC and CRC IEC, compared to control and CD patients. $\mathrm{F}_{\mathrm{sp}}$ from all donors was calculated; there is an overall significant difference in IL-13Ra 1 and IL-13Ra 2 protein expression among the four groups studied $(\mathrm{p}<0.0001$ and $\mathrm{p}=0.0078$, respectively; Figure 2). We detected very low levels of IL-13Ra 1 expression on control and CD IEC, while its expression is significantly higher in UC and CRC IEC. Expression of IL-13Ra1 is also significantly higher in CRC IEC ( $p \leq 0.05$ ) compared to UC. There is significant up-regulation of IL-13Ra2 on CRC IEC compared to control, CD, and UC 
donors, and on UC IEC compared to control and CD donors. While IL-4Ra expression is low in all four groups, there is a significant difference in its expression among the groups (p $=0.05$ ), and in UC compared to that of control, $\mathrm{CD}$, and colon cancer patients.

These results were confirmed using an alternate immunoblot approach. Increased expression of both the IL-13Ra 1 and IL-13Ra 2 receptor chains is observed in all UC patients examined compared to control and CD donors (Fig. 2D), which agrees with the flow cytometric analysis. Furthermore, CRC IEC show increased expression of IL-13Ra1 and IL-13Ra 2 chains compared to non-transformed IEC from the same patient, which acts as an internal control. No reproducible change in expression of the IL-4Ra receptor chain in the different categories of patients and in control donors was observed.

\section{IL-13 signaling in IEC from diseased and control donors through the canonical STAT6 pathway and alternative MAPK pathways are markedly distinct}

As IL-13Ra 1 expression is increased on UC and CRC IEC, we investigated the robustness of IL-13 signaling through STAT6 phosphorylation (pSTAT6) (15) in freshly isolated IEC from control, inflamed, and transformed tissue stimulated with $50 \mathrm{ng} / \mathrm{ml}$ of IL-13 for various times (Figure 3). IL-13 induced similar STAT6 phosphorylation in control and CRC IEC, while the kinetics of the STAT6 response in inflamed CD IEC is somewhat delayed. Despite increased IL-13Ra 1 expression on UC IEC, IL-13 stimulated no detectable pSTAT6. Due to the inherent tendency of IEC to undergo apoptosis (21), viability of these cells was determined to be $>95 \%$ after 30 min stimulation with IL-13 (data not shown).

Since there is now one report that IL-13 activates an AP-1 variant containing c-jun and Fra-2 (20), we also investigated the activation of MAPK pathways by IL-13 in control and diseased IEC, especially in light of the differences in IL-13Ra 1 and IL-13Ra 2 subunit expression. In non-transformed control and CD IEC, expressing low amounts of IL-13Ra 1 and IL-13Ra2, IL-13 induced ERK 1/2 phosphorylation within 5 min, which rapidly declined by $10 \mathrm{~min}$ (Figure 3). In UC and CRC IEC, which express higher amounts of IL-13Ra 1 and IL-13Ra2, pERK 1/2 is present at an elevated basal level, and IL-13 does not induce a further increase in ERK 1/2 activation. Similarly, JNK and p38 phosphorylation is induced by IL-13 within 2 and 5 min, respectively, in control and CD IEC, but IL-13 is unable to activate JNK or p38 in UC and CRC IEC, despite increased expression of the IL-13Ra 1 and IL-13Ra 2 chains. Densitometry analysis in four consecutive donors from each group confirmed these unique activation profiles for STAT6 and MAPK, while demonstrating no change in total STAT6 or MAP kinase expression (data not shown). Together these results suggest that when IL-13Ra2 is expressed at low surface density, IL-13 signals through both the STAT6 and MAPK pathways. It is possible that as cell surface expression of IL-13Ra 2 increases, IL-13 signaling through STAT6 and MAPK pathways may be inhibited, confirming the current belief that increased IL-13Ra2 expression inhibits IL-13 signaling (19). However, in CRC IEC with the highest expression of IL-13Ra2, only MAPK signaling is blocked. Since CRC IEC also express high amounts of IL-13Ra1, the net effect may be to reach an equilibrium that enables STAT6 signaling.

\section{IL-13Ra1 and IL-4Ra are dispensable for IL-13-induced signals through the MAPK pathways}

To identify the relationship between receptor expression and signal transduction, neutralizing antibodies to IL-13Ra 1 and IL-4Ra were used to inhibit IL-13 signaling in a human colon-derived cell line HT-29.19A, which express medium and low amounts of $\operatorname{IL}-13 \operatorname{Ra} 1\left(\mathrm{~F}_{\mathrm{sp}}=27.67\right)$ and IL-13Ra2 $\left(\mathrm{F}_{\mathrm{sp}}=4.1\right)$, comparable to the levels seen in control and CD IEC (Figure 4A). In the presence of anti-IL-13Ra 1 and anti-IL-4Ra alone or together, IL-13 signaling through the STAT6 pathway was blocked, but IL-13-induced ERK 
1/2, JNK, and p38 phosphorylation was unaffected (Figure 4B). These results indicate that neither the IL-13Ra 1 nor the IL-4Ra receptor subunits participate in IL-13 signal transduction through any of the MAPK pathways. Thus a likely candidate mediating IL-13 activation of ERK 1/2, JNK, and p38 is the IL-13Ra2 chain.

\section{Expression of the IL-13Ra2 receptor chain is critical for ERK activation, yet extremely elevated IL-13Ra2 expression inhibits STAT6 and ERK activation}

To test our hypothesis that IL-13Ra2 supports MAPK signaling, while high concentrations inhibit this pathway, we developed a cell culture model in which IL-13Ra2 levels were varied. HT-29.19A cells were differentiated in the presence of IL-13 and TNF- $a$ for $72 \mathrm{~h}$ to generate graded amounts of cell surface expression of the IL-13Ra2 receptor chain. Cytokine-treated and untreated cells expressing various concentrations of IL-13Ra2 were isolated by cell sorting. Six populations of cells were prepared (Figure 5): (i) unsorted parental HT-29.19A expressing low amounts of IL-13Ra2, (ii) parental and (iii) cytokinetreated HT-29.19A that express no detectable IL-13Ra2, and cytokine-treated HT-29.19A expressing (iv) low, (v) medium, and (vi) high amounts of IL-13Ra2. No change in IL-13Ra1 expression was observed in these various cell populations, and IL-13 activated both the STAT6 and ERK 1/2 pathways in parental HT-29.19A cells (Fig 5A). Our findings from five separate experiments show that in cells expressing no detectable IL-13Ra2, IL-13 still signals through STAT6 pathway, but not through the ERK pathway (Fig. 5B), consistent with our conclusion that IL-13Ra1 initiates the STAT6 pathway (Fig. 4) and that the MAPK pathways originate with IL-13Ra2. In keeping with the proposed, alternate role of IL-13Ra 2 as a decoy receptor; as the surface density of the IL-13Ra 2 chain gradually increases, the intensity of IL-13-induced STAT6 and ERK activation declines (Fig. 5C and $5 \mathrm{D})$.

\section{Reversal of IL-13Ra2 inhibition of both STAT6 and ERK signaling by high agonist exposure}

The dual function for high affinity IL-13Ra2 proposes that at low surface density this protein signals through the MAPK pathway, while at high concentrations the chain acts as a reservoir for IL-13 and blocks all responses. To test this hypothesis and extend the observation to a second cell lineage, we investigated transfectants of the monocytic cell line U937 that express graded concentrations of IL-13Ra2 (24). Expression of IL-13Ra 1 and IL-13Ra 2 was confirmed by flow cytometry in untransfected, parental U937 cells (low surface expression of IL-13Ra2) and the stable transfectants (Figure 6). Comparable to HT-29.19A cells, parental U937 signals through the STAT6 and ERK pathways at low and high IL-13 concentrations. If higher concentrations of IL-13Ra 2 compete with IL-13Ra 1 as a means to block signal transduction, we predict that in cells expressing inhibitory levels of IL-13Ra2, elevated IL-13 should reverse this inhibition. Low concentrations of IL-13 (10 $\mathrm{ng} / \mathrm{ml}$ ) in U937 transfectants expressing elevated amounts of IL-13Ra2 were unable to stimulate STAT6 phosphorylation, while ERK signaling was intact. An excess of IL-13 (200 $\mathrm{ng} / \mathrm{ml}$ ) activated both the STAT6 and ERK pathways, consistent with our proposal that IL-13Ra 1 and IL-13Ra 2 compete for IL-13 binding. Thus, the character and intensity of an IL-13 response can be modulated in two ways: by the target cell, which can vary the ratio of receptor chain expression, and by the level of IL-13 secreted from the neighboring lymphocyte.

\section{Discussion}

UC-associated CRC is one of many examples where the inflammatory process acts as a cofactor for carcinogenesis (25). One popular view on the association of UC and cancer proposes that inflammatory signaling within epithelial cells can lead to inappropriate 
survival and transformation. An alternative model involves an indirect mechanism, in which inflammation of the mucosa, induced by contact with intestinal microflora, promotes oncogenesis of the overlying epithelium (26). An extension of this indirect model is likely to implicate cytokines, which function as mediators and regulators of the inflammatory response (27), play a pathogenic role in IBD, and target both hematopoietic and stromal cells at the site of inflammation. Mucosal inflammation in UC is partially driven by increased secretion of IL-13 produced by NKT cells, which manifest reactivity to antigens presented by epithelial cells (8). Similarly, tissue damage in the oxazolone-induced model of colitis results from induction of natural killer T (NKT) cells that produce IL-13 (7). We reported in the IL-10 ${ }^{\text {null }}$ model of colitis that after 20 weeks of colonic inflammation, local expression of IL-13 is increased (6), and this is accompanied by epithelial dysplasia. Furthermore, inhibition of IL-13 activity partially reverses inflammation (28) and prevents dysplasia (data not shown).

The functional defects of UC and CRC IEC may be directly attributed to the effects of IL-13 on epithelial cells. IL-13 is an important effector cytokine in UC that impairs epithelial barrier function by affecting epithelial apoptosis, tight junctions, and restitution velocity (29). In HT-29 CL.16E cells, which express all three IL-13 receptor chains, IL-13 markedly increased expression of the goblet cell products trefoil factor family peptides (TFF3) and mucin (MUC2) (11). IL-13Ra1 is upregulated in human keratinocytes from psoriatic skin (30), and in Hodgkins lymphoma where IL-13 acts as an autocrine growth factor (31). IL-13Ra 2 expression has been demonstrated in numerous tissues, including murine spleen and brain, and human liver, lung, and thymus (18). Tumor cell lines originating from malignant glioma (32), squamous cell carcinoma of the head and neck (33) express large numbers of IL-13Ra2. The striking increase in both IL-13Ra 1 and IL-13Ra 2 receptor chains in the UC and CRC epithelium is consistent with elevated receptor expression at sites of inflammation and tumorigenesis, and provides one potential link between UC and CRC.

The biological effects of IL-13 are thought to be mediated by a shared receptor composed of the IL-4Ra and IL-13Ra 1 chains (10), and a decoy receptor attributed to the high affinity IL-13Ra2 protein (15). In the present study we report that IL-13Ra 1 and IL-13Ra2 expression is significantly elevated in UC and CRC IEC, compared to control and CD cells, which is consistent with our hypothesis that IL-13 might promote epithelial cell oncogenesis in UC-associated CRC. Furthermore, our results illustrate differential signaling induced by IL-13 in UC and CRC IEC when contrasted with control and CD cells. In particular, IL-13 induces STAT6 phosphorylation in control, CD, and CRC IEC, but not in UC. On the surface this finding conflicts with the significant increase in IL-13 receptor chain expression in UC and suggests a modification of IL-13 function in the UC epithelium. STAT-mediated signal transduction plays a pivotal role in IBD inflammatory processes, which are characterized by a mucosal cytokine imbalance $(9,34)$. STAT6 expression and activity are perturbed in a variety of malignancies, including colorectal cancer (35). For example, STAT6 signaling is activated in Hodgkin's lymphoma (36) and prostate cancer (37), suggesting that active STAT6 signaling may be beneficial for cancer cell growth. Downregulation of STAT6 in a prostate cancer cell line resulted in reduced cell viability, induction of apoptosis, reduced Bcl-2 and Bcl-xl expression, and impaired migration (37). STAT6 signaling is also able to regulate cell growth, proliferation, and apoptosis in a colon cancer cell line (38).

Our study also reveals that the MAPK pathways are not induced by IL-13 in UC and CRC IEC, providing further evidence that IL-13-mediated functions are altered in these disease states. IL-13 signaling through MAPK is widely attributed to the IL-13a 1 chain (39), although we demonstrate that in the absence of IL-13Ra 1 function or expression, ERK 1/2, JNK, and p38 phosphorylation is maintained. In fact, low to medium surface expression of 
IL-13Ra2 supports signaling through the MAPK pathway. The MAPK signaling cascades respond to diverse extracellular stimuli that initiate inflammatory cellular responses (40) and also function as major proliferative signals in many tumor cells, including colorectal cancers (41). IL-13 activates MAPK pathways in a variety of epithelial cell types (42). The p38 MAPK pathway is involved in IL-13-induced mucus cell metaplasia in mouse tracheal epithelial cells (43). IL-13-mediated changes in goblet cell density and MUC5a expression in bronchial epithelial cell cultures are regulated by MAPK (42), and IL-13 modulation of the transition of human lung fibroblasts to myofibroblasts is dependent on the JNK pathway (44). Thus our results indicate that IL-13 activates the MAPK pathway via the IL-13Ra2 chain, which may modulate epithelial cell proliferation and apoptosis during $\mathrm{UC}$ and the transformation associated with CRC.

In summary, we report that high expression of IL-13a 2 inhibits both STAT6 and ERK signaling. Yet, contrary to previous studies we conclude that IL-13Ra2 is not only a decoy receptor, but instead responds to IL-13 and biases the cell toward enhanced MAPK signaling. Furthermore, the inhibitory effect of IL-13Ra 2 is reversed by high concentrations of IL-13 or a proportionate increase in IL-13Ra1. Our results invoke the following biochemical explanation. When IL-13Ra2 is expressed at a low to moderate cell surface density, it functions alone, or may heterodimerize with an unreported receptor chain, to signal through the MAPK pathways, thus competing equally with IL-13Ra 1 for binding to IL-13. As the surface expression of IL-13Ra2 increases dramatically, as in UC, the equilibrium is shifted toward homodimerization, yielding the classic high affinity decoy receptor that competes effectively to quench both IL-13Ra 1- and IL-13Ra2-mediated signal transduction. If, however, expression of IL-13Ra 1 and IL-13Ra 2 increase coordinately, as in CRC, then IL-13Ra 1 can compete effectively for IL-13 binding and signal through the STAT6 pathway, while IL-13Ra2 remains homodimerized and is unable to signal.

\section{Acknowledgments}

We thank Dr. P. Fu of the Department of Epidemiology and Biostatics for statistical analysis.

Support: This research was supported by the Tissue Procurement and Histology Core Facility of the Comprehensive Cancer Center of Case Western Reserve University and University Hospitals Case Medical Center (NIH/NCI P30 CA43703), and by grants from the National Institute of Health (DK-54213 and AI-53188) and the American Cancer Society, Ohio Division, Inc. to A.D.L.

\section{References}

1. Chambers WM, Warren BF, Jewell DP, et al. Cancer surveillance in ulcerative colitis. Br J Surg. 2005; 92:928-936. [PubMed: 16034807]

2. Eaden JA, Abrams KR, Mayberry JF. The risk of colorectal cancer in ulcerative colitis: a metaanalysis. Gut. 2001; 48:526-535. [PubMed: 11247898]

3. Mantovani A, Allavena P, Sica A, et al. Cancer-related inflammation. Nature. 2008; 454:436-444. [PubMed: 18650914]

4. Berg DJ, Davidson N, Kuhn R, et al. Enterocolitis and colon cancer in interleukin-10-deficient mice are associated with aberrant cytokine production and CD4(+) TH1-like responses. J Clin Invest. 1996; 98:1010-1020. [PubMed: 8770874]

5. Rudolph U, Finegold MJ, Rich SS, et al. Ulcerative colitis and adenocarcinoma of the colon in G alpha i2-deficient mice. Nat Genet. 1995; 10:143-150. [PubMed: 7663509]

6. Spencer DM, Veldman GM, Banerjee S, et al. Distinct inflammatory mechanisms mediate early versus late colitis in mice. Gastroenterology. 2002; 122:94-105. [PubMed: 11781285]

7. Heller F, Fuss IJ, Nieuwenhuis EE, et al. Oxazolone colitis, a Th2 colitis model resembling ulcerative colitis, is mediated by IL-13-producing NK-T cells. Immunity. 2002; 17:629-638. [PubMed: 12433369] 
8. Fuss IJ, Heller F, Boirivant M, et al. Nonclassical CD1d-restricted NK T cells that produce IL-13 characterize an atypical Th2 response in ulcerative colitis. J Clin Invest. 2004; 113:1490-1497. [PubMed: 15146247]

9. Bouma G, Strober W. The immunological and genetic basis of inflammatory bowel disease. Nat Rev Immunol. 2003; 3:521-533. [PubMed: 12876555]

10. Wynn TA. IL-13 effector functions. Annu Rev Immunol. 2003; 21:425-456. [PubMed: 12615888]

11. Blanchard C, Durual S, Estienne M, et al. IL-4 and IL-13 up-regulate intestinal trefoil factor expression: requirement for STAT6 and de novo protein synthesis. J Immunol. 2004; 172:37753783. [PubMed: 15004182]

12. Kanai T, Watanabe M, Hayashi A, et al. Regulatory effect of interleukin-4 and interleukin-13 on colon cancer cell adhesion. Br J Cancer. 2000; 82:1717-1723. [PubMed: 10817509]

13. Trejdosiewicz LK, Morton R, Yang Y, et al. Interleukins 4 and 13 upregulate expression of cd44 in human colonic epithelial cell lines. Cytokine. 1998; 10:756-765. [PubMed: 9811528]

14. Endo Y, Marusawa H, Kou T, et al. Activation-Induced Cytidine Deaminase Links Between Inflammation and the Development of Colitis-Associated Colorectal Cancers. Gastroenterology. 2008

15. Kelly-Welch A, Hanson EM, Keegan AD. Interleukin-13 (IL-13) pathway. Sci STKE. 2005; 2005:cm8. [PubMed: 16030286]

16. Obiri NI, Leland P, Murata T, et al. The IL-13 receptor structure differs on various cell types and may share more than one component with IL-4 receptor. J Immunol. 1997; 158:756-764. [PubMed: 8992992]

17. Miloux B, Laurent P, Bonnin O, et al. Cloning of the human IL-13R alpha1 chain and reconstitution with the IL4R alpha of a functional IL-4/IL-13 receptor complex. FEBS Lett. 1997; 401:163-166. [PubMed: 9013879]

18. Donaldson DD, Whitters MJ, Fitz LJ, et al. The murine IL-13 receptor alpha 2: molecular cloning, characterization, and comparison with murine IL-13 receptor alpha 1. J Immunol. 1998; 161:2317-2324. [PubMed: 9725226]

19. Wood N, Whitters MJ, Jacobson BA, et al. Enhanced interleukin (IL)-13 responses in mice lacking IL-13 receptor alpha 2. J Exp Med. 2003; 197:703-709. [PubMed: 12642602]

20. Fichtner-Feigl S, Strober W, Kawakami K, et al. IL-13 signaling through the IL-13alpha2 receptor is involved in induction of TGF-beta1 production and fibrosis. Nat Med. 2006; 12:99-106. [PubMed: 16327802]

21. Grossmann J, Artinger M, Grasso AW, et al. Hierarchical cleavage of focal adhesion kinase by caspases alters signal transduction during apoptosis of intestinal epithelial cells. Gastroenterology. 2001; 120:79-88. [PubMed: 11208716]

22. Kedar E, Ikejiri BL, Bonnard GD, et al. A rapid technique for isolation of viable tumor cells from solid tumors: use of the tumor cells for induction and measurement of cell-mediated cytotoxic responses. Eur J Cancer Clin Oncol. 1982; 18:991-1000. [PubMed: 6984388]

23. Das L, Levine AD. TGF-beta inhibits IL-2 production and promotes cell cycle arrest in TCRactivated effector/memory $\mathrm{T}$ cells in the presence of sustained TCR signal transduction. $\mathrm{J}$ Immunol. 2008; 180:1490-1498. [PubMed: 18209044]

24. Daines MO, Tabata Y, Walker BA, et al. Level of expression of IL-13R alpha 2 impacts receptor distribution and IL-13 signaling. J Immunol. 2006; 176:7495-7501. [PubMed: 16751396]

25. Balkwill F, Mantovani A. Inflammation and cancer: back to Virchow? Lancet. 2001; 357:539-545. [PubMed: 11229684]

26. Clevers H. At the crossroads of inflammation and cancer. Cell. 2004; 118:671-674. [PubMed: 15369667]

27. Germano G, Allavena P, Mantovani A. Cytokines as a key component of cancer-related inflammation. Cytokine. 2008; 43:374-379. [PubMed: 18701317]

28. Spencer DM, Levine AD. Neutralizing IL-4 or IL-13 alters mucosal immunity in late phase colitis of IL-10-/- mice. FASEB J. 2003; 17:C-230.

29. Heller F, Florian P, Bojarski C, et al. Interleukin-13 is the key effector Th2 cytokine in ulcerative colitis that affects epithelial tight junctions, apoptosis, and cell restitution. Gastroenterology. 2005; 129:550-564. [PubMed: 16083712] 
30. Wongpiyabovorn J, Suto H, Ushio H, et al. Up-regulation of interleukin-13 receptor alpha1 on human keratinocytes in the skin of psoriasis and atopic dermatitis. J Dermatol Sci. 2003; 33:3140. [PubMed: 14527737]

31. Ohshima K, Akaiwa M, Umeshita R, et al. Interleukin-13 and interleukin-13 receptor in Hodgkin's disease: possible autocrine mechanism and involvement in fibrosis. Histopathology. 2001; 38:368375. [PubMed: 11318902]

32. Mintz A, Gibo DM, Slagle-Webb B, et al. IL-13Ralpha2 is a glioma-restricted receptor for interleukin-13. Neoplasia. 2002; 4:388-399. [PubMed: 12192597]

33. Kawakami M, Kawakami K, Kasperbauer JL, et al. Interleukin-13 receptor alpha 2 chain in human head and neck cancer serves as a unique diagnostic marker. Clin Cancer Res. 2003; 9:6381-6388. [PubMed: 14695138]

34. Mudter J, Weigmann B, Bartsch B, et al. Activation pattern of signal transducers and activators of transcription (STAT) factors in inflammatory bowel diseases. Am J Gastroenterol. 2005; 100:6472. [PubMed: 15654782]

35. Klampfer L. The role of signal transducers and activators of transcription in colon cancer. Front Biosci. 2008; 13:2888-2899. [PubMed: 17981761]

36. Skinnider BF, Elia AJ, Gascoyne RD, et al. Signal transducer and activator of transcription 6 is frequently activated in Hodgkin and Reed-Sternberg cells of Hodgkin lymphoma. Blood. 2002; 99:618-626. [PubMed: 11781246]

37. Das S, Roth CP, Wasson LM, et al. Signal transducer and activator of transcription-6 (STAT6) is a constitutively expressed survival factor in human prostate cancer. Prostate. 2007; 67:1550-1564. [PubMed: 17705178]

38. Zhang M, Zhou Y, Xie C, et al. STAT6 specific shRNA inhibits proliferation and induces apoptosis in colon cancer HT-29 cells. Cancer Lett. 2006; 243:38-46. [PubMed: 16387423]

39. David M, Ford D, Bertoglio J, et al. Induction of the IL-13 receptor alpha2-chain by IL-4 and IL-13 in human keratinocytes: involvement of STAT6, ERK and p38 MAPK pathways. Oncogene. 2001; 20:6660-6668. [PubMed: 11709700]

40. Van Den Blink B, Ten Hove T, Van Den Brink GR, et al. From extracellular to intracellular targets, inhibiting MAP kinases in treatment of Crohn's disease. Ann N Y Acad Sci. 2002; 973:349-358. [PubMed: 12485892]

41. Fang JY, Richardson BC. The MAPK signalling pathways and colorectal cancer. Lancet Oncol. 2005; 6:322-327. [PubMed: 15863380]

42. Atherton HC, Jones G, Danahay H. IL-13-induced changes in the goblet cell density of human bronchial epithelial cell cultures: MAP kinase and phosphatidylinositol 3-kinase regulation. Am J Physiol Lung Cell Mol Physiol. 2003; 285:L730-739. [PubMed: 12794003]

43. Fujisawa T, Ide K, Holtzman MJ, et al. Involvement of the p38 MAPK pathway in IL-13-induced mucous cell metaplasia in mouse tracheal epithelial cells. Respirology. 2008; 13:191-202. [PubMed: 18339016]

44. Hashimoto S, Gon Y, Takeshita I, et al. IL-4 and IL-13 induce myofibroblastic phenotype of human lung fibroblasts through c-Jun NH2-terminal kinase-dependent pathway. J Allergy Clin Immunol. 2001; 107:1001-1008. [PubMed: 11398077]

\section{Abbreviations}

$\begin{array}{ll}\text { CRC } & \text { Colorectal Cancer } \\ \text { CD } & \text { Crohn's Disease } \\ \text { IEC } & \text { Intestinal epithelial cells } \\ \text { IBD } & \text { Inflammatory Bowel Disease } \\ \text { IL-13 } & \text { Interleukin 13 } \\ \text { LPMC } & \text { Lamina propria mononuclear cell }\end{array}$


MAPK Mitogen Activated Protein Kinase

STAT6 Signal Transducer and activator of transcription 6

UC Ulcerative colitis 
$\mathrm{IL}-13 \mathrm{R} \alpha 1$
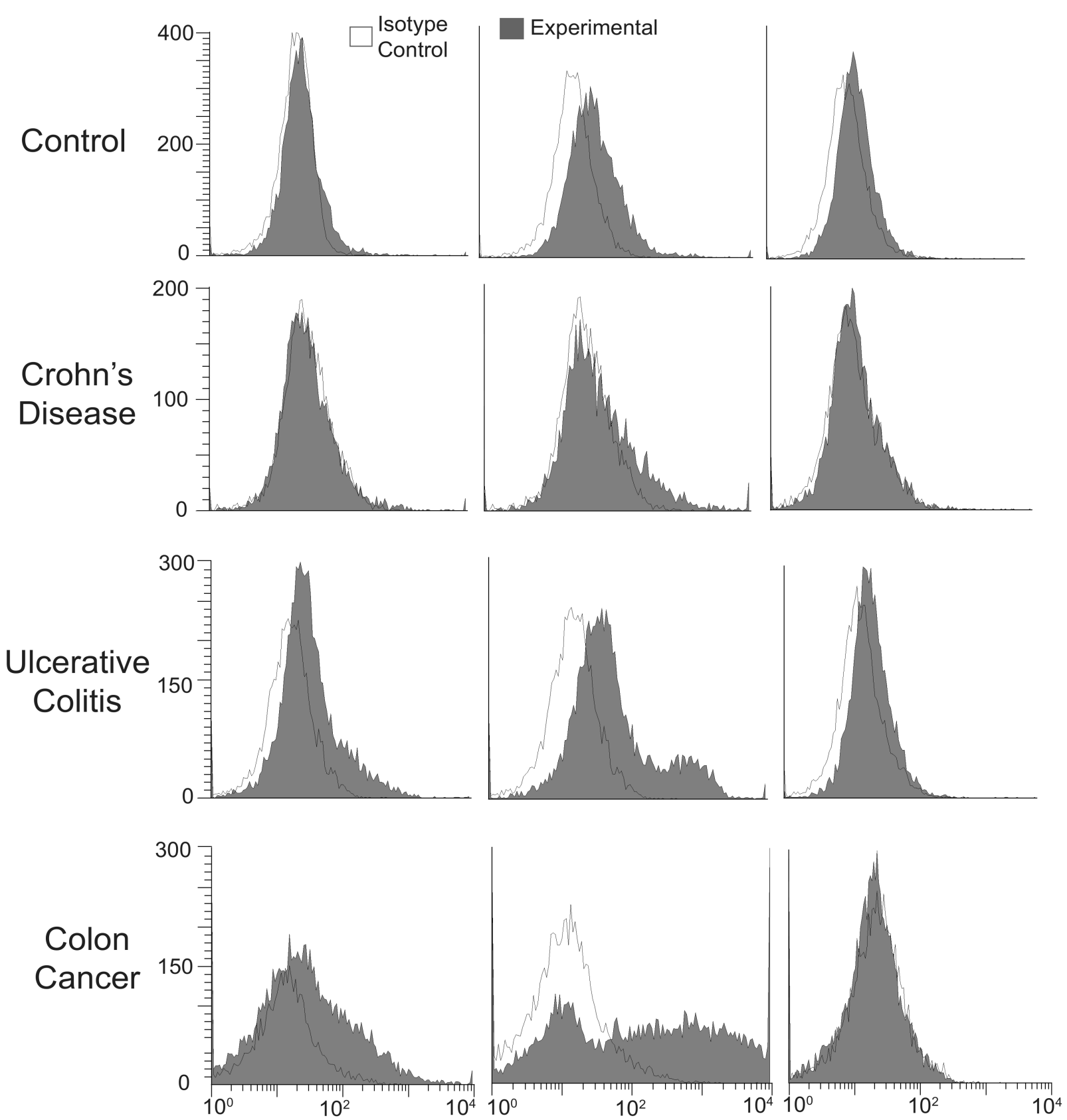

Figure 1. Increased expression of IL-13Ra1 and IL-13Ra2 on IEC from colon cancer and ulcerative colitis patients

IEC from healthy regions of control donors and diseased lesions from patients were indirectly immunostained with PE for surface expression of three IL-13 receptor chains (grey histograms) and analyzed by flow cytometry: IL-13Ra1 (left panels), IL-13Ra2 (middle panels) and IL-4Ra (right panels). White, open histograms represent isotype control. Representative immunofluorescence histograms from a total of 6 control, $4 \mathrm{CD}, 5$ $\mathrm{UC}$ and 3 CRC patients are shown. 
A

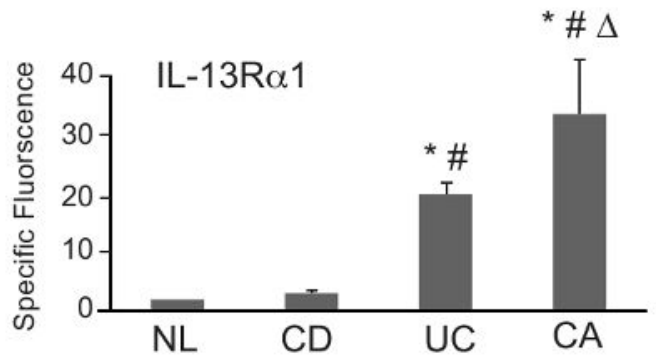

B

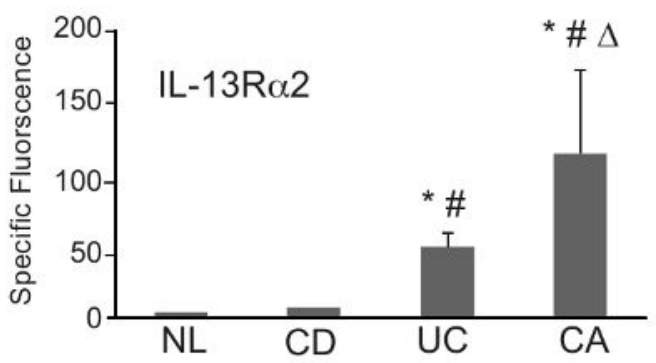

C

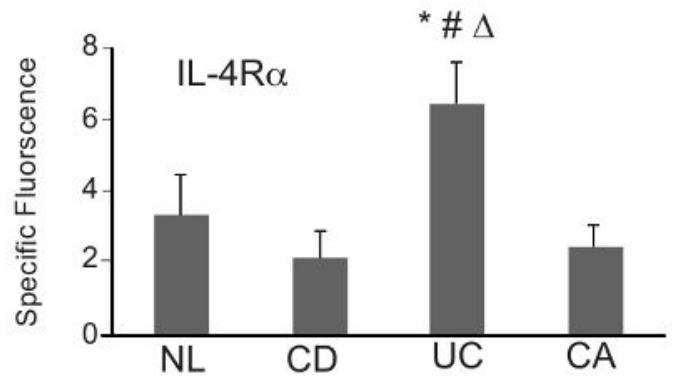

D
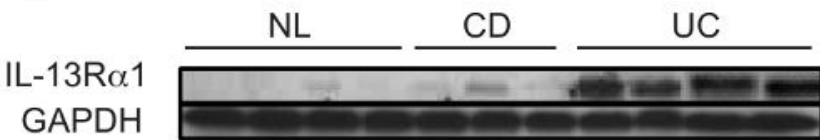

NL CA NL CA

GAPDH

$$
\begin{array}{r}
\text { IL-13R } \alpha 2 \\
\text { GAPDH }
\end{array}
$$

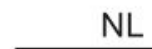

CD

UC

-

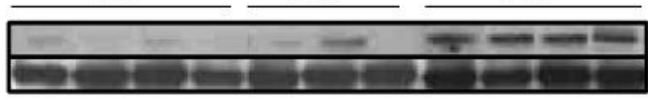

NL CA NL CA

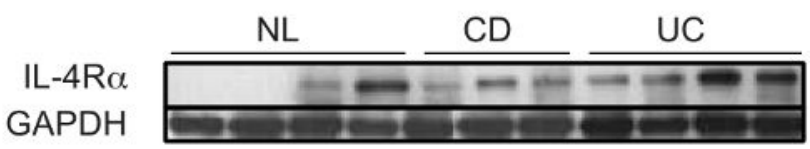

एक

NL CA NL CA

GAPDH

Figure 2. Significant increase in IL-13Ra1 and IL-13Ra2 surface protein expressio $\mathrm{n}$ on IEC from UC and CRC lesions

$\mathrm{F}_{\mathrm{sp}}$ were calculated from 6 control, $4 \mathrm{CD}, 5 \mathrm{UC}$, and $3 \mathrm{CRC}$ tumor samples. A) IL-13Ra 1 expression: * $\mathrm{p} \leq 0.05$ for UC and CRC compared with control, ${ }^{*} \mathrm{p} \leq 0.05$ for UC and CRC compared to $\mathrm{CD},{ }^{\Delta} \mathrm{p} \leq 0.05$ for CRC compared to UC. B) IL-13Ra 2 expression: *p $\leq 0.05$ for CRC compared to control, ${ }^{\#} \mathrm{p} \leq 0.05$ for CRC compared to $\mathrm{CD},{ }^{\Delta} \mathrm{p} \leq 0.05$ for CRC compared to UC. C) IL-4Ra expression: * $\mathrm{p} \leq 0.05$ for UC compared to control, ${ }^{*} \mathrm{p} \leq 0.05$ for UC compared to $\mathrm{CD},{ }^{\Delta} \mathrm{p} \leq 0.05$ for UC compared to CA. D) IEC cells from healthy control, CD, UC and CRC were isolated and whole cell extracts prepared. Extract normalized for total protein concentration, fractioned by SDS-PAGE and immunoblotted 
with antibodies directed against IL-13Ra 1, IL-13Ra2, and IL-4Ra. GAPDH loading control is shown. 
A

Control

B CD
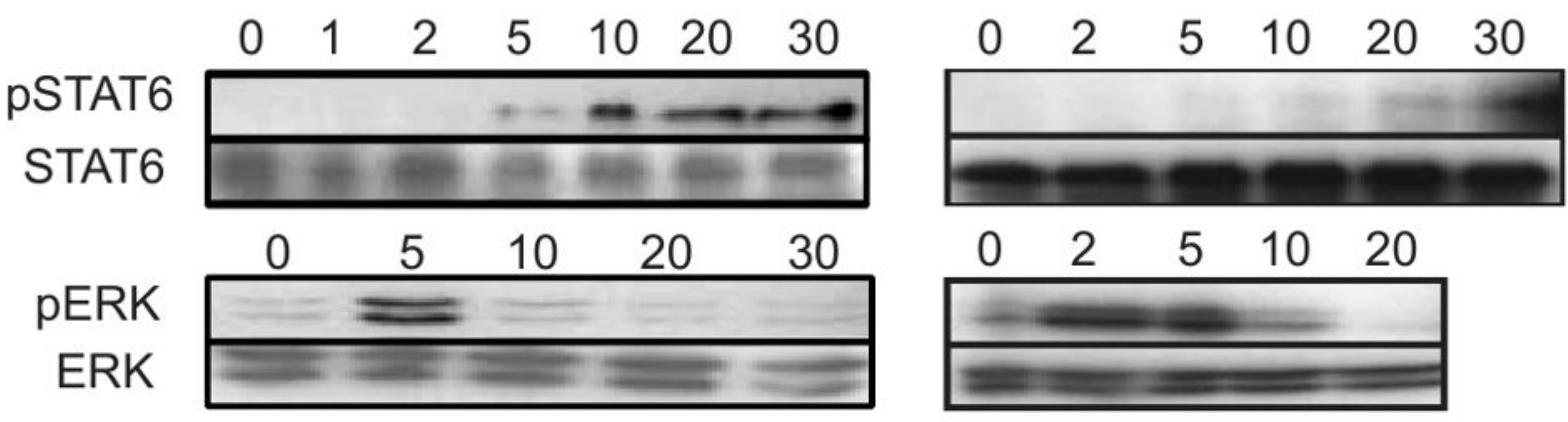

pJNK
JNK
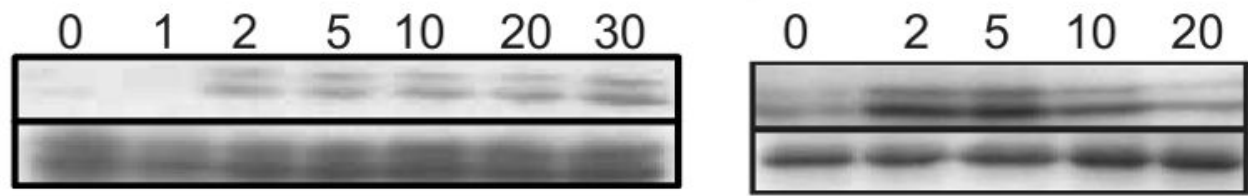

pp38

p38
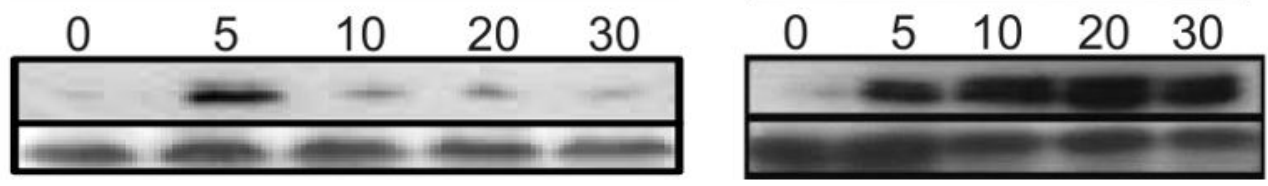

C

UC

D CA
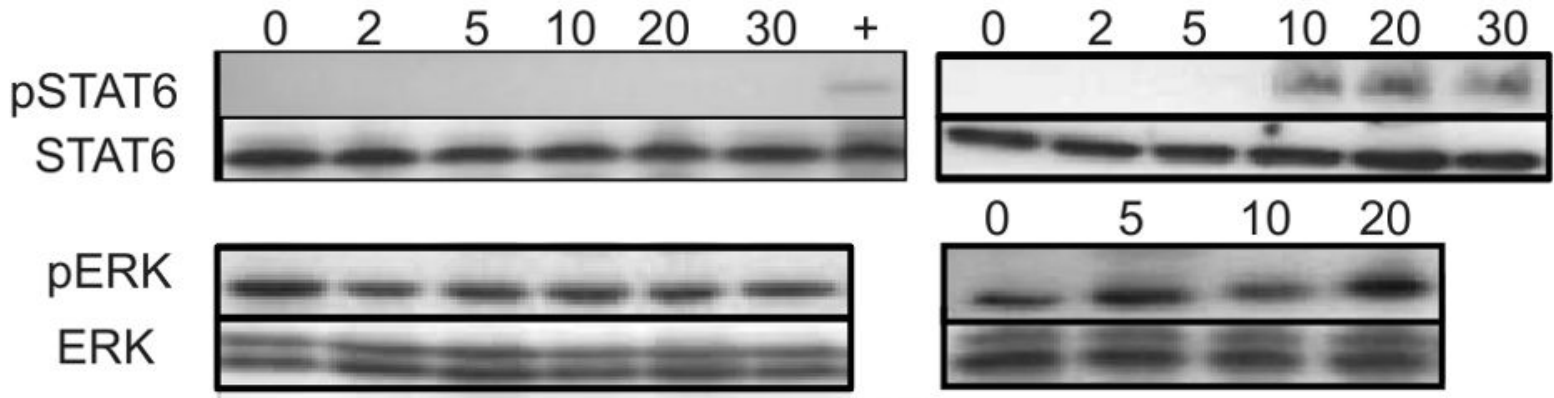

pJNK

JNK
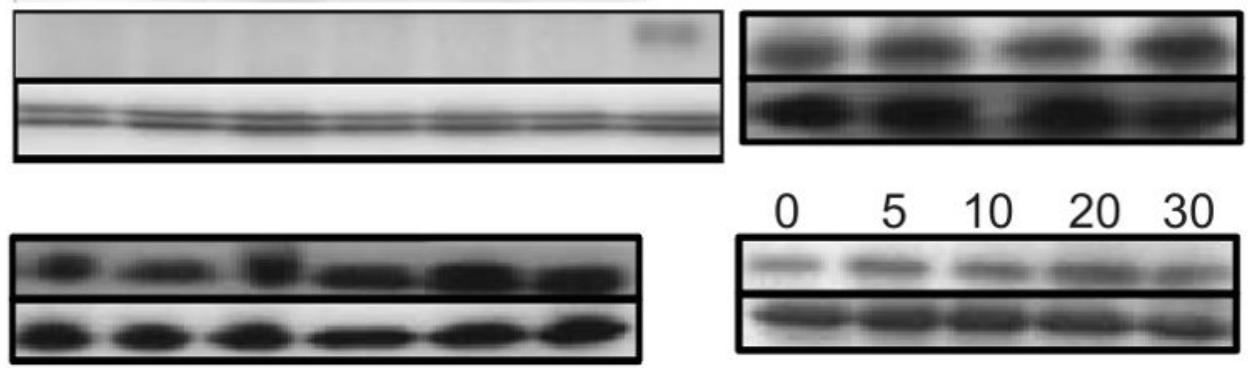

Figure 3. IEC from UC and CRC donors do not transduce a signal through the ERK, JNK, and p38 pathways after IL-13 exposure

Freshly isolated IEC from A) unaffected regions, B) CD inflamed, C) UC inflamed, and D) colon tumors were rested for $4 \mathrm{~h}$ and stimulated with $50 \mathrm{ng} / \mathrm{ml} \mathrm{IL-13.} \mathrm{Total} \mathrm{cellular} \mathrm{proteins}$ were extracted from untreated and treated cells after various times $(1,2,5,10,20$, or $30 \mathrm{~min}$ depending on number of available cells) and fractionated by SDS-PAGE. Phosphorylated and total STAT6, ERK 1/2, JNK, and p38 were detected by immunoblot. Data shown is representative of 4 control, $3 \mathrm{CD}, 3 \mathrm{UC}$, and 4 colon tumor samples. 

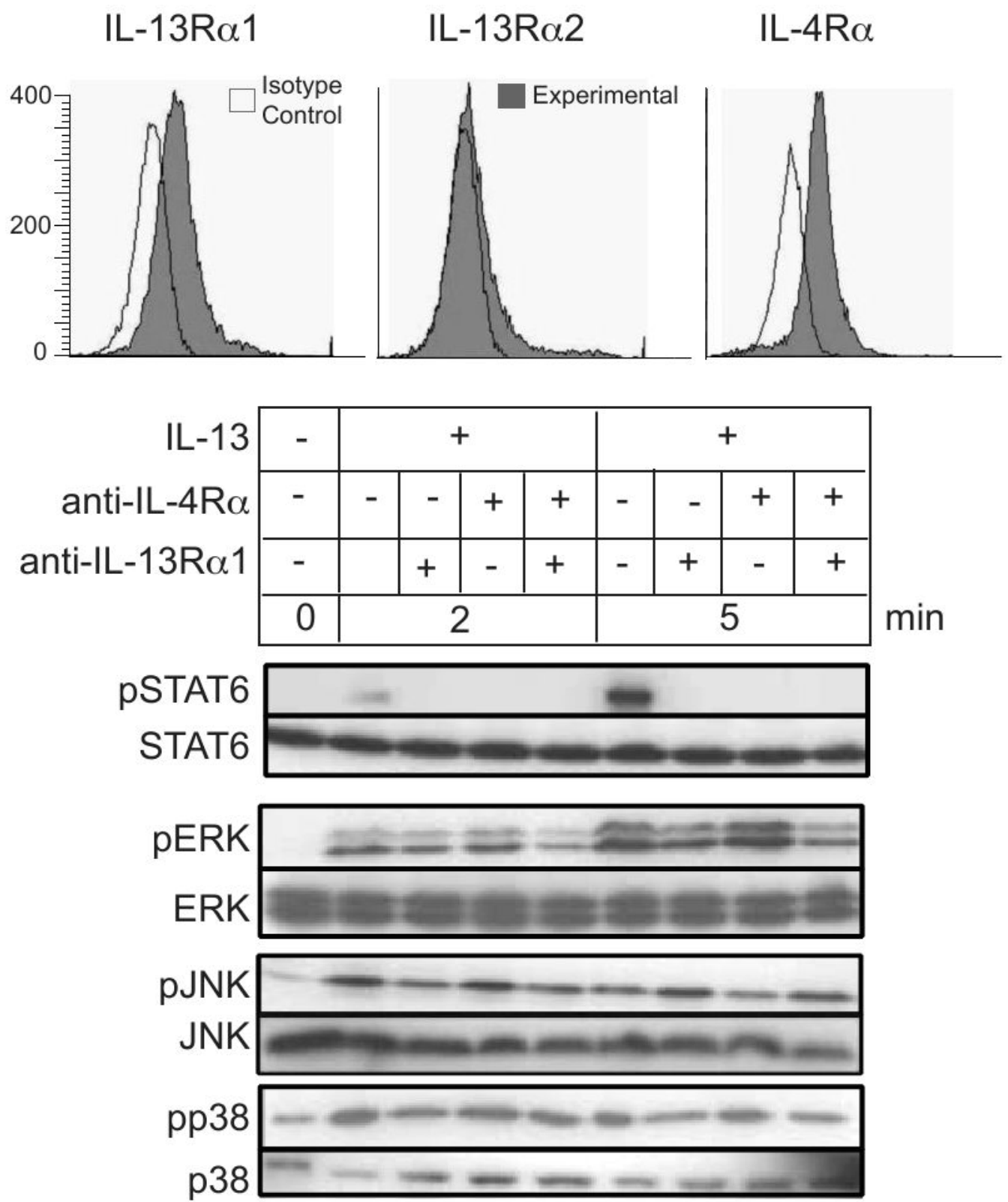

Figure 4. Neutralizing the IL-13Ra1 and IL-4Ra chains inhibits IL-13-induced signaling through STAT6, but not through ERK, JNK, and p38

HT-29.19A cells were rested for $4 \mathrm{~h}$ pre-incubated with either anti-IL-13Ra 1 or antiIL-4Ra, or both for $1 \mathrm{~h}$ at $37^{\circ} \mathrm{C}$ and then stimulated with $20 \mathrm{ng} / \mathrm{ml} \mathrm{IL-13}$ for 2 and $5 \mathrm{~min}$. Whole cell extracts were fractioned by SDS-PAGE and immunoblotted for phospho-specific and total anti-STAT6, ERK, JNK, and p38. Data is representative of two experiments. 

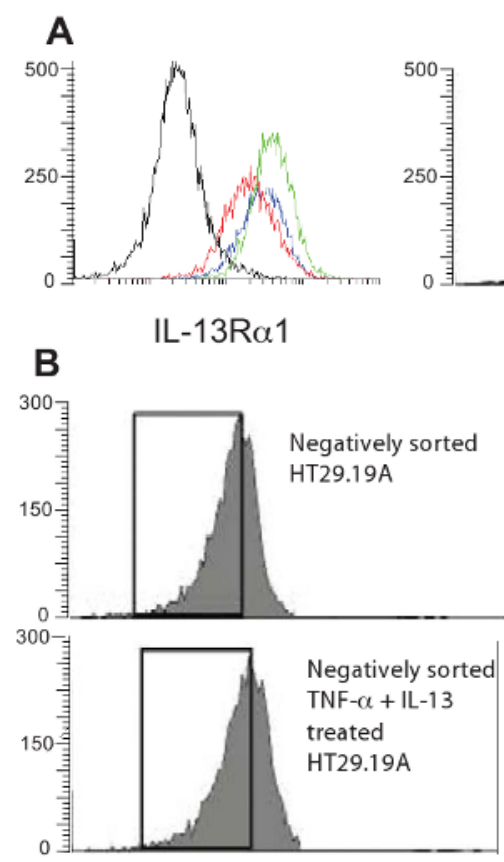

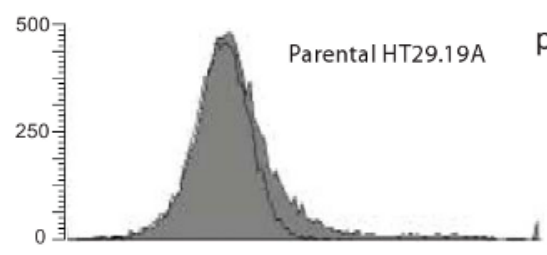

IL-13R $\alpha 2$
IL-13: $0 \quad 5$ min

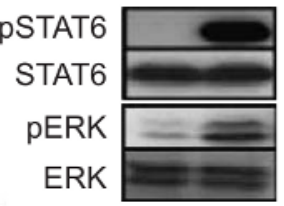

IL-13: $0 \quad 5$ min

pSTAT6

STAT6

pERK

ERK
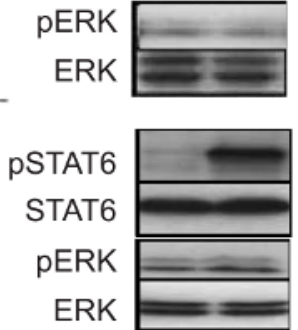

C

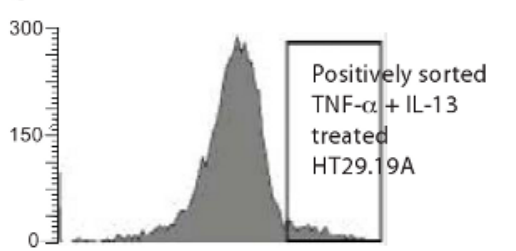

IL-13: $0 \quad 5 \mathrm{~min}$

pSTAT6

STAT6

pERK

ERK

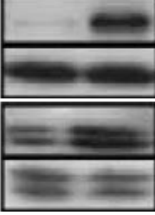

300 - Positively sorted

TNF- $\alpha+$ IL-13

treated

150 童

HT29.19A

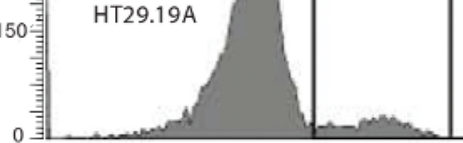

pSTAT6

STAT6

pERK

ERK
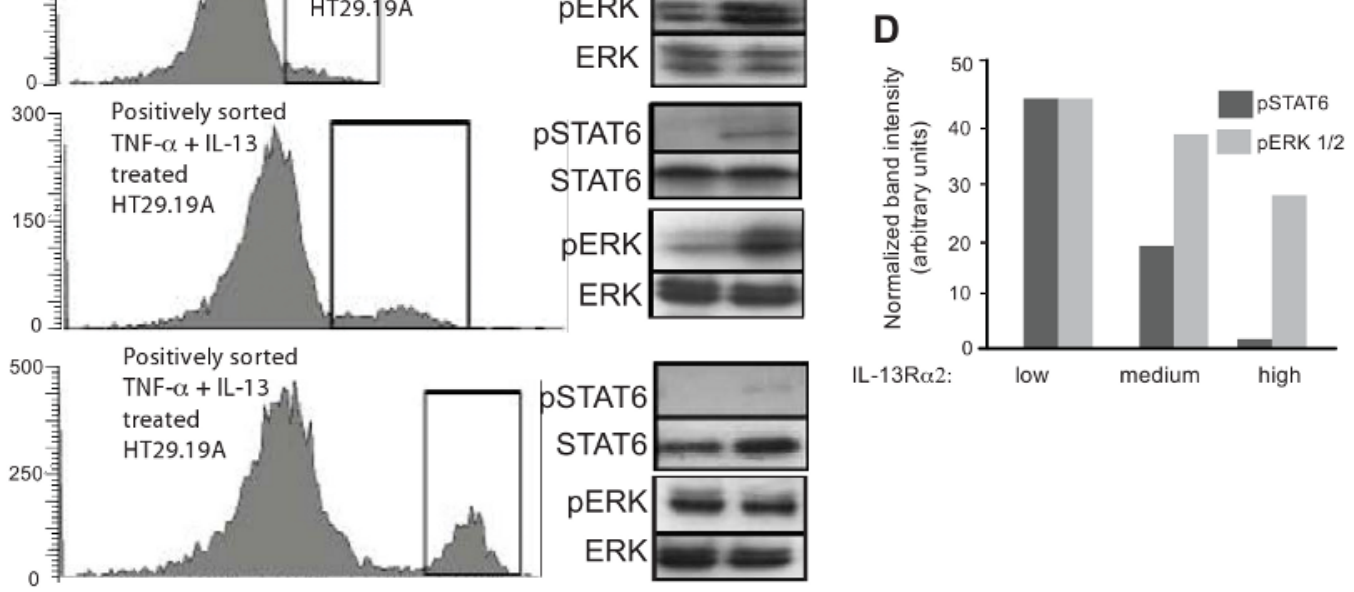

Figure 5. Low IL-13Ra2 expression facilitates ERK activation, while high expression inhibits STAT6

HT-29.19A cells were treated with IL-13 and TNF-a for $72 \mathrm{~h}$ and sorted for IL-13Ra2 expression by flow cytometry generating six groups. Cells were stimulated with $50 \mathrm{ng} / \mathrm{ml}$ IL-13. Total cellular proteins were extracted from unstimulated and stimulated cells at 5 min, fractionated by SDS-PAGE, and probed for phospho-specific and total STAT6 and ERK. (Panel A) Untreated or cytokine-treated HT29.19A cells were indirectly immunostained for surface expression of IL-13Ra 1 and analyzed by flow cytometry. Isotype control staining is shown in black, parental HT29.19A in red, and the two cytokinetreated cultures in green and blue. Unsorted parental HT29.19A express low amounts of 
IL-13Ra2. (Panel B) Negatively sorted, untreated or cytokine-treated HT29.19A with no detectable IL-13Ra2 expression. (Panel C) Cytokine-treated, positively sorted HT29.19A for low, medium, or high amounts of IL-13Ra2. (Panel D) Densitometric quantization of the phosphorylated forms of STAT6 and ERK 1/2 normalized to total STAT6 and ERK 1/2 at 5 min of IL-13 stimulation. 
A

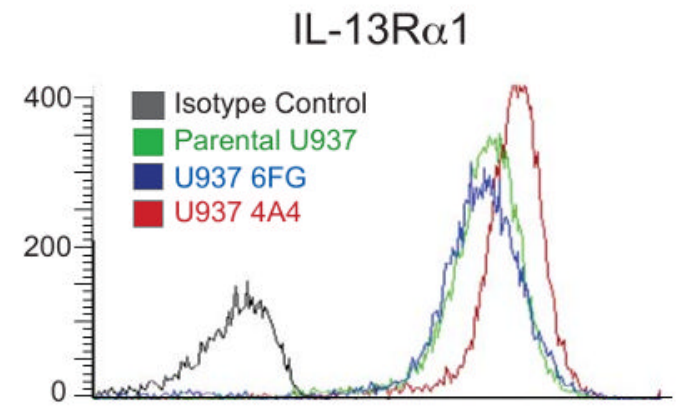

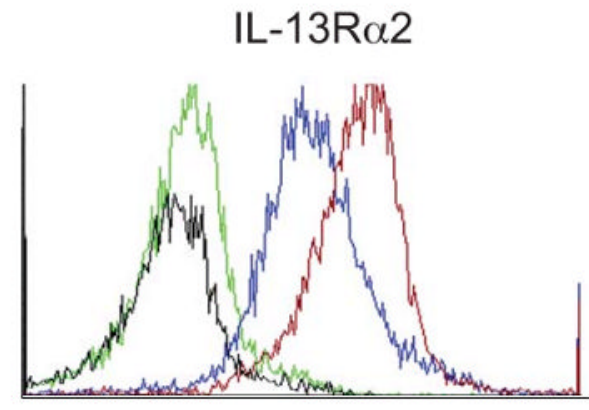

IL-13 200 (ng/ml)
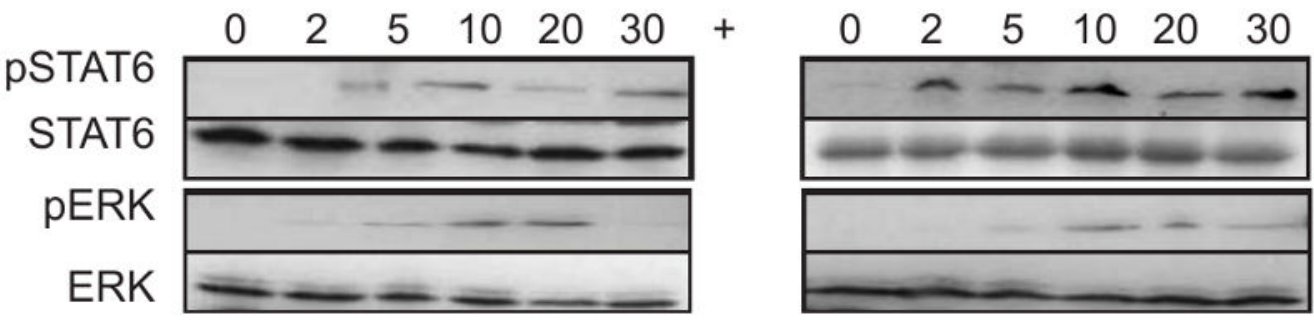

$\min$

B

U937

C

U937 6FG

\section{pSTAT6 \\ STAT6}

pERK

ERK
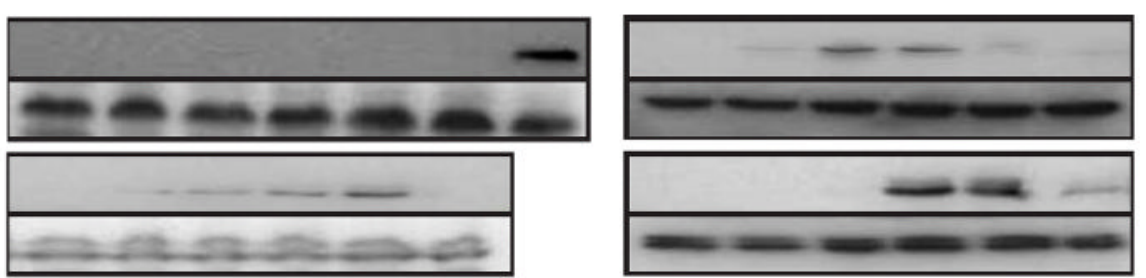

D

U937 4A4
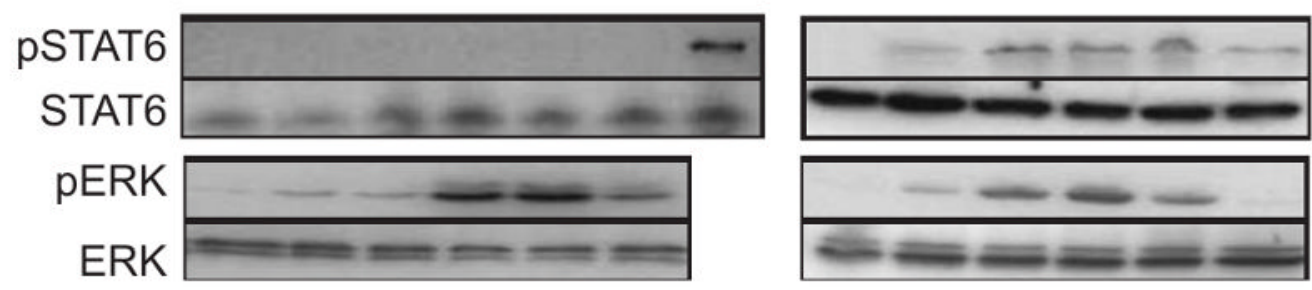

Figure 6. Reversal of decoy function of IL-13Ra2 by increased concentrations of IL-13

A) Parental and IL-13Ra2-transfected U937 cells were analyzed by flow cytometry for IL-13Ra 1 and IL-13Ra 2 expression. Isotype control staining is shown with the black lines, parental U937 in green, and the two transfectants in red and blue. B) U937 cell lines were stimulated with IL-13 (10 ng/ml and $200 \mathrm{ng} / \mathrm{ml})$, and total cellular proteins were extracted, fractionated by SDS-PAGE, and probed for phospho-specific and total STAT6 and ERK expression. The lane marked (+) is an extract of parental U937 cells stimulated with $50 \mathrm{ng} /$ $\mathrm{ml}$ IL-13 for 5 min to mark the presence of a pSTAT6 signal on membranes containing extracts in which STAT was not phosphorylated. 